\title{
PLANETARY PHASE VARIATIONS OF THE 55 CANCRI SYSTEM
}

\author{
Stephen R. Kane ${ }^{1}$, Dawn M. Gelino ${ }^{1}$, David R. Ciardi ${ }^{1}$, Diana Dragomir ${ }^{1,2}$, and Kaspar von Braun ${ }^{1}$ \\ ${ }^{1}$ NASA Exoplanet Science Institute, Caltech, MS 100-22, 770 South Wilson Avenue, Pasadena, CA 91125, USA; skane@ipac.caltech.edu \\ ${ }^{2}$ Department of Physics \& Astronomy, University of British Columbia, Vancouver, BC V6T1Z1, Canada \\ Received 2011 May 6; accepted 2011 July 21; published 2011 September 29
}

\begin{abstract}
Characterization of the composition, surface properties, and atmospheric conditions of exoplanets is a rapidly progressing field as the data to study such aspects become more accessible. Bright targets, such as the multi-planet 55 Cancri system, allow an opportunity to achieve high signal-to-noise for the detection of photometric phase variations to constrain the planetary albedos. The recent discovery that innermost planet, 55 Cancri e, transits the host star introduces new prospects for studying this system. Here we calculate photometric phase curves at optical wavelengths for the system with varying assumptions for the surface and atmospheric properties of 55 Cancri e. We show that the large differences in geometric albedo allows one to distinguish between various surface models, that the scattering phase function cannot be constrained with foreseeable data, and that planet $\mathrm{b}$ will contribute significantly to the phase variation, depending upon the surface of planet e. We discuss detection limits and how these models may be used with future instrumentation to further characterize these planets and distinguish between various assumptions regarding surface conditions.
\end{abstract}

Key words: planetary systems - stars: individual (55 Cancri) - techniques: photometric

\section{INTRODUCTION}

The discovery of transiting exoplanets was a breakthrough in the exoplanet field. This is not simply because it provided an additional avenue through which to secure their detection, but because of the vast amount of planetary characterization information they yield access to. The investment in improving photometric techniques and precision for transit surveys has led to the peripheral consequence of detecting more subtle planetary signatures. Photometric phase curves of exoplanets are one such signature, though technically difficult to detect due to the relatively low signal amplitudes. Examples of observed phase variations in the infrared (IR) from Spitzer observations include HD 189733b (Knutson et al. 2009a) and HD 149026b (Knutson et al. 2009b). Examples in the optical include Kepler observations of HAT-P-7b (Welsh et al. 2010) and phase variations detected in the light curve of CoRoT-1b (Snellen et al. 2009).

The most valuable transiting planets are the ones that orbit bright host stars because they lend themselves toward greater signal-to-noise measurements. Follow-up of known radial velocity (RV) planets is a key way to achieve this goal (Kane et al. 2009). A recent example is that of the planets orbiting 55 Cancri (HD 75732, HIP 43587, HR 3522, hereafter 55 Cnc), a bright $(V=5.95)$ G8 dwarf star. Valenti \& Fischer (2005) predict a relatively old age for the $55 \mathrm{Cnc}$ system of $9.5 \pm 4.4 \mathrm{Gyr}$, although Fischer et al. (2008) estimate a more modest age range of 2-8 Gyr. Thus this is an interesting system since the host is of similar spectral type and age to our own sun and yet the planetary configuration is substantially different from our own system.

The first planet in the system was discovered by Butler et al. (1997) and the second and third were detected by Marcy et al. (2002). The fourth Neptune-mass planet, $55 \mathrm{Cnc}$ e, was found by McArthur et al. (2004), with an originally deduced orbital period of 2.82 days. The fifth planet was discovered by Fischer et al. (2008), leaving us with the currently known five-planet system. Subsequent study of the aliases in the power spectrum of the RV data by Dawson \& Fabrycky (2010) found that the true period of the e planet most likely 0.74 days rather than 2.82 days. This was confirmed by Winn et al. (2011) who successfully detected the transit of 55 Cnc e using high-precision photometry from the Microvariability and Oscillations of Stars (MOST) satellite (Walker et al. 2003). Further confirmation appeared through detection of the transit in the IR via Spitzer observations (Demory et al. 2011a).

This system presents an opportunity to search for phase variations for a known system around a very bright star where we know much more about the relative planetary inclinations in the system than other similar multi-planet systems. Phase variations for gas giants have been described by Kane \& Gelino (2010) and Kane \& Gelino (2011) and the albedos and heat redistribution properties for such planets are discussed by Cowan \& Agol (2011). Here we apply these techniques to model the phase variations of the $55 \mathrm{Cnc}$ system using the revised orbital parameters. Winn et al. (2011) report the detection of phase modulations that are inconsistent with their expectations, though more phase coverage to improve the amplitude measurement and account for the presence of the outer planets would assist in their interpretation. The flux ratio of a planet with radius $R_{p}$ to the host star measured at wavelength $\lambda$ is defined as

$$
\epsilon(\alpha, \lambda) \equiv \frac{f_{p}(\alpha, \lambda)}{f_{\star}(\lambda)}=A_{g}(\lambda) g(\alpha, \lambda) \frac{R_{p}^{2}}{r^{2}}
$$

where $\alpha$ is the phase angle of the planet. This flux ratio consists of three major components; the geometric albedo $A_{g}(\lambda)$, the phase function $g(\alpha, \lambda)$, and the inverse-square relation to the star-planet separation $r$. In Section 3 we describe the system characteristics which are used as input into the flux ratio model for each of the planets. In Section 4 we calculate the total system phase variations based upon three different models of the inner planet. Finally, in Section 5, we assess the detectability of the individual and combined planetary signals and future prospects for discriminating between the surface models for the e planet. 
Table 1

Planetary Orbital Parameters and Derived Characteristics

\begin{tabular}{|c|c|c|c|c|c|c|c|c|c|c|c|}
\hline \multirow[t]{2}{*}{ Planet } & \multirow{2}{*}{$\begin{array}{c}P^{\mathrm{a}} \\
\text { (days) }\end{array}$} & \multirow{2}{*}{$\begin{array}{c}M_{p} \sin i^{\mathrm{a}} \\
\left(M_{J}\right)\end{array}$} & \multirow{2}{*}{$\begin{array}{c}a^{\mathrm{a}} \\
(\mathrm{AU})\end{array}$} & \multirow[t]{2}{*}{$e^{\mathrm{a}}$} & \multirow{2}{*}{$\begin{array}{c}\omega^{\mathrm{a}} \\
(\operatorname{deg})\end{array}$} & \multirow{2}{*}{$\begin{array}{l}R_{p}^{\mathrm{b}} \\
\left(R_{J}\right)\end{array}$} & \multirow{2}{*}{$\begin{array}{c}i \\
(\operatorname{deg})\end{array}$} & \multirow[t]{2}{*}{$A_{g}{ }^{\mathrm{c}}$} & \multicolumn{3}{|c|}{ Flux Ratio $\left(10^{-6}\right)$} \\
\hline & & & & & & & & & Rocky & Molten & Atmosphere \\
\hline $\mathrm{e}$ & 0.736537 & 0.026 & 0.016 & 0.17 & 181 & 0.18 & 90.0 & 0.15 & 3.37 & 20.23 & 4.89 \\
\hline b & 14.6507 & 0.825 & 0.115 & 0.010 & 139 & 1.10 & 87.5 & 0.16 & $\ldots$ & $\ldots$ & 3.26 \\
\hline $\mathrm{c}$ & 44.364 & 0.171 & 0.240 & 0.005 & 252 & 1.01 & 88.8 & 0.17 & $\ldots$ & $\ldots$ & 0.70 \\
\hline $\mathrm{f}$ & 259.8 & 0.155 & 0.781 & 0.30 & 180 & 0.96 & 89.6 & 0.22 & $\ldots$ & $\ldots$ & 0.11 \\
\hline $\mathrm{d}$ & 5169.0 & 3.82 & 5.74 & 0.014 & 186 & 1.07 & 89.9 & 0.50 & $\ldots$ & $\ldots$ & 0.004 \\
\hline
\end{tabular}

Notes.

${ }^{\text {a }}$ From Table 10 of Dawson \& Fabrycky (2010).

b With the exception of planet e, based upon Bodenheimer et al. (2003) models.

${ }^{c}$ Mean geometric albedo assuming a thick atmosphere.

\section{SCIENCE MOTIVATION}

Planetary phase variations are currently difficult to detect due to their relatively low amplitude. It is reasonable, therefore, to assess the science return from such challenging observations. For planets that undergo secondary eclipses, the albedo may be determined through careful modeling of the eclipse data (see, for example, Deming et al. 2011). This has the distinctive advantage of being able to measure the key planetary parameters of radius and orbital inclination; however, without complete phase variation analysis, this albedo determination is generally only valid at or near the substellar point of the planet. Albedos are sensitive to such aspects as cloud formation depth, reflective condensates in the upper atmosphere, and the scattering properties of the surface/atmosphere. Thus there is a strong degeneracy between the albedo and back-scattering properties when the planet is only observed at zero phase angle. By determining the phase function through precision observations, one may gain further insight into these aspects, which are inaccessible via secondary eclipse observations.

Furthermore, continued observations of the phase function contain information on the combined reflective properties for all planets in the system. For planets whose presence and/or orbital properties are unknown, this can lead to ambiguity in the interpretation of the data (Kane \& Gelino 2010). However, in cases such as the $55 \mathrm{Cnc}$ system, the planets presence and orbital parameters are well determined and thus the degeneracy can be removed leading to constraints on the scattering properties or the outer planets. As we shall demonstrate, planet $b$ contributes significantly to the total phase variation in this case. Even if the planets are not known to transit, the phase curves can be used to constrain the inclination of the orbits (Kane \& Gelino 2011).

\section{SYSTEM CHARACTERISTICS}

Here we describe the system characteristics that are used as input for the phase model, both measured and derived. These are summarized in Table 1, along with the predicted phase variation amplitudes which are described in more detail in Section 4.

\subsection{Orbital Considerations}

We adopt the complete system orbital solution shown in Table 10 of Dawson \& Fabrycky (2010). This is a self-consistent model which converges on the solution which includes the correct period for planet e of 0.74 days. The fit also has a smaller rms scatter of the residuals than the equivalent fit, which reaches the old e period of 2.82 days. These orbital properties are shown in Table 1 , including the period $P$, the minimum mass $M_{p} \sin i$, the semimajor axis $a$, the eccentricity $e$, and the argument of periastron $\omega$. Note that the phase models presented here also account for the eccentricities present in the orbits. For the host star, we adopt a stellar mass of $M_{\star}=0.94 \pm 0.05 M_{\odot}$ (Fischer et al. 2008) and a stellar radius of $R_{\star}=0.943 \pm 0.010 R_{\odot}$ (von Braun et al. 2011).

\subsection{Planetary Radii}

An important property for considering the amplitude of the planet-to-star flux ratio from a given planet is the planetary radius. This quantity is normally only available for a planet whose transits reveal it to us, but can also be derived from the estimated planetary mass and stellar properties for the planet in question. For planet e, we adopt the radius measured by Winn et al. (2011) of $2.00 R_{\oplus}$, or $0.179 R_{J}$. We select this radius rather than that measured by Demory et al. (2011a) because we are considering phase variation effects at passbands similar to MOST rather than Spitzer. For the more massive outer planets in the system, we calculate radii estimates based upon the models of Bodenheimer et al. (2003), which take into account both the planetary mass and the stellar flux received at their respective semimajor axis. The results of these calculations are shown in Table 1.

\subsection{Orbital Inclinations}

The orbital inclinations of the planets are unknown except for that of planet e. We adopt the value of $i=90^{\circ}$ for this planet from Winn et al. (2011). The evidence thus far is that none of the outer planets transit the host star. Here we assume that this is indeed the case and calculate the maximum inclination which satisfies this criteria using the methods described in Kane \& von Braun (2008). The inclination in this case is then given by

$$
\cos i=\frac{R_{p}+R_{\star}}{r}
$$

where $r$ is the star-planet separation, given by

$$
r=\frac{a\left(1-e^{2}\right)}{1+e \cos f}
$$

and is evaluated at $\omega+f=\pi / 2$.

The above assumption for the maximum inclination hinges somewhat on the system orbits being close to coplanar. Astrometry performed by McArthur et al. (2004) indicate that planet $d$ may be misaligned with the edge-on orbit of planet e, although this is based upon preliminary work and the outermost planet 
contributes a negligible amount of flux to the combined phase curve. The results from the Kepler mission have revealed many multiple-transiting systems, which implies that those systems are remarkably coplanar (Latham et al. 2011). An excellent example is the six-planet system orbiting Kepler-11 (Lissauer et al. 2011). Theoretical modeling performed by Tremaine \& Dong (2011) independently supports the claim by Lissauer et al. (2011) that near-zero mutual inclinations are favored for multiplanet systems. Stability analyses of RV systems have also been used to determine coplanarity, such as the cases of the HD 10180 system (Lovis et al. 2011) and GJ 876 system (Bean \& Seifahrt 2009). It should be noted however that dynamical stability over long timescales can be achieved through interaction of giant planets or the influence of an external perturber (Guillochon et al. 2011; Malmberg et al. 2002).

\subsection{Geometric Albedos}

Planetary albedos can span a very large range of values depending upon both the surface conditions and location of the planet. The theoretical models of Sudarsky et al. (2005) show that there is a dependence of gas giant geometric albedos on star-planet separation due to the removal of reflective condensates from the upper atmosphere. This was quantified by Kane \& Gelino (2010) who also generalized this dependence to eccentric orbits. Note that this does not take into account a variable surface albedo or the thermal response of the surface/ atmosphere to changing incident flux. We use these models to estimate the geometric albedos for the four outermost planets.

However, the super-Earth planet e is a special case due to its smaller size and proximity to the host star. In Section 4 we consider three possible surface models, which entail different albedos and scattering properties. The models are that of a rocky surface, a molten surface, and a thick atmosphere. The atmosphere model uses the geometric albedos described above with the same star-planet separation dependence. Table 1 shows the mean calculated albedos for all of the planets based upon the thick atmosphere assumption. The rocky and molten surface models use the measured albedos of Mercury and Io as templates, respectively, where the values were extracted from the JPL HORIZONS System. ${ }^{3}$ Note that the composition and tidal forces on Io lead to a highly variable surface albedo (Simonelli et al. 2001). However, we are considering the integrated flux from the planet and so the mean albedo of Io is a useful approximation. Mercury's geometric albedo is 0.106 and has a density of $5.427 \mathrm{~g} \mathrm{~cm}^{-3}$. For Io, the geometric albedo is 0.6 and the density is $3.530 \mathrm{~g} \mathrm{~cm}^{-3}$. The density for $55 \mathrm{Cnc}$ e is calculated from the properties in Table 1 to be $5.6 \mathrm{~g} \mathrm{~cm}^{-3}$. This is comparable to that of Mercury and Io although the reflective properties at the surface are independent of the composition. Additionally, the similar bulk density to Mercury implies a greater concentration of volatiles resulting in high densities at the core with relatively low-density near the surface.

\subsection{Variable Incident Stellar Flux}

The geometric albedo can have a spatial dependence, which is due to a variable amount of stellar flux being received at various points on the planetary surface. Consider the cases of Mercury and Venus. Mercury experiences a large range of dayside surface temperatures due to a steep temperature gradient between the equator and the poles. The 3:2 spin-orbit resonance of Mercury

\footnotetext{
3 http://ssd.jpl.nasa.gov/?horizons
}

results in the periodic cooling off of the surface as it crosses the terminator into the night-side of the planet. In the case of Venus, the slow retrograde rotation would also allow for extreme temperature gradients both on the dayside and at the day/night boundary if it were not for the thick atmosphere, which is exceptionally efficient at redistributing the trapped thermal radiation.

From the planetary radius and semimajor axis for $55 \mathrm{Cnc}$ e shown in Table 1, the stellar flux at the orbital distance of the planetary poles is $\sim 10 \%$ less than the flux at the equator. Spreading this flux onto the surface of the planet at a given latitude then yields a surface flux which is further reduced by the cosine of the angle the host star is from zenith at that location. However, we assume that the planet is tidally locked such that no cooling of the dayside surface ever occurs. For the various surface models described in Section 4 we then consider a constant albedo for the dayside surface. For the molten surface model in particular, the variable stellar flux is sufficient at all latitude to result in the necessary sustained temperatures for melting the surface silicate materials. This is described further in Section 4.

\section{PHASE VARIATIONS}

Here we simulate the phase variations of the $55 \mathrm{Cnc}$ system. The phase algorithms are based upon the formalism of Kane $\&$ Gelino (2010). For each planet, the phase angle $\alpha$ is defined to be zero at superior conjunction and is described by

$$
\cos \alpha=\sin (\omega+f) \sin i,
$$

where $f$ is the true anomaly and $i$ is the inclination of the orbit. The phase function of a Lambert sphere assumes isotropic scattering of incident flux over $2 \pi$ sr and is described by

$$
g(\alpha, \lambda)=\frac{\sin \alpha+(\pi-\alpha) \cos \alpha}{\pi}
$$

and is used for the rocky and molten surface models described below. For the atmosphere model, we adopt the empirically derived phase function of Hilton (1992), which is based upon observations of Jupiter and Venus. This approach contains a correction to the planetary visual magnitude of the form

$$
\Delta m(\alpha)=0.09\left(\alpha / 100^{\circ}\right)+2.39\left(\alpha / 100^{\circ}\right)^{2}-0.65\left(\alpha / 100^{\circ}\right)^{3}
$$

leading to a phase function given by

$$
g(\alpha)=10^{-0.4 \Delta m(\alpha)},
$$

which we refer to as the Hilton phase function and allows for non-isotropic (cloud) scattering. Here we confine our study to optical wavelengths centered on $550 \mathrm{~nm}$. This places the study near the peak response of the Kepler and MOST detectors. It is possible for there to be a thermal component to the phase variation at these wavelengths, such as that predicted by the models of Demory et al. (2011b) for Kepler-7b. In addition, Welsh et al. (2010) speculate that the Kepler observations of HAT-P-7b may include a thermal component due to the planet not emitting as a blackbody as is often assumed. Depending on the specific surface scattering properties, this may lead to a non-negligible underestimate of the phase amplitude for the e planet that will be interesting to resolve when more precision photometry is acquired. Note that studies of flux ratio dependencies on wavelength have been undertaken by Sudarsky et al. (2005). 

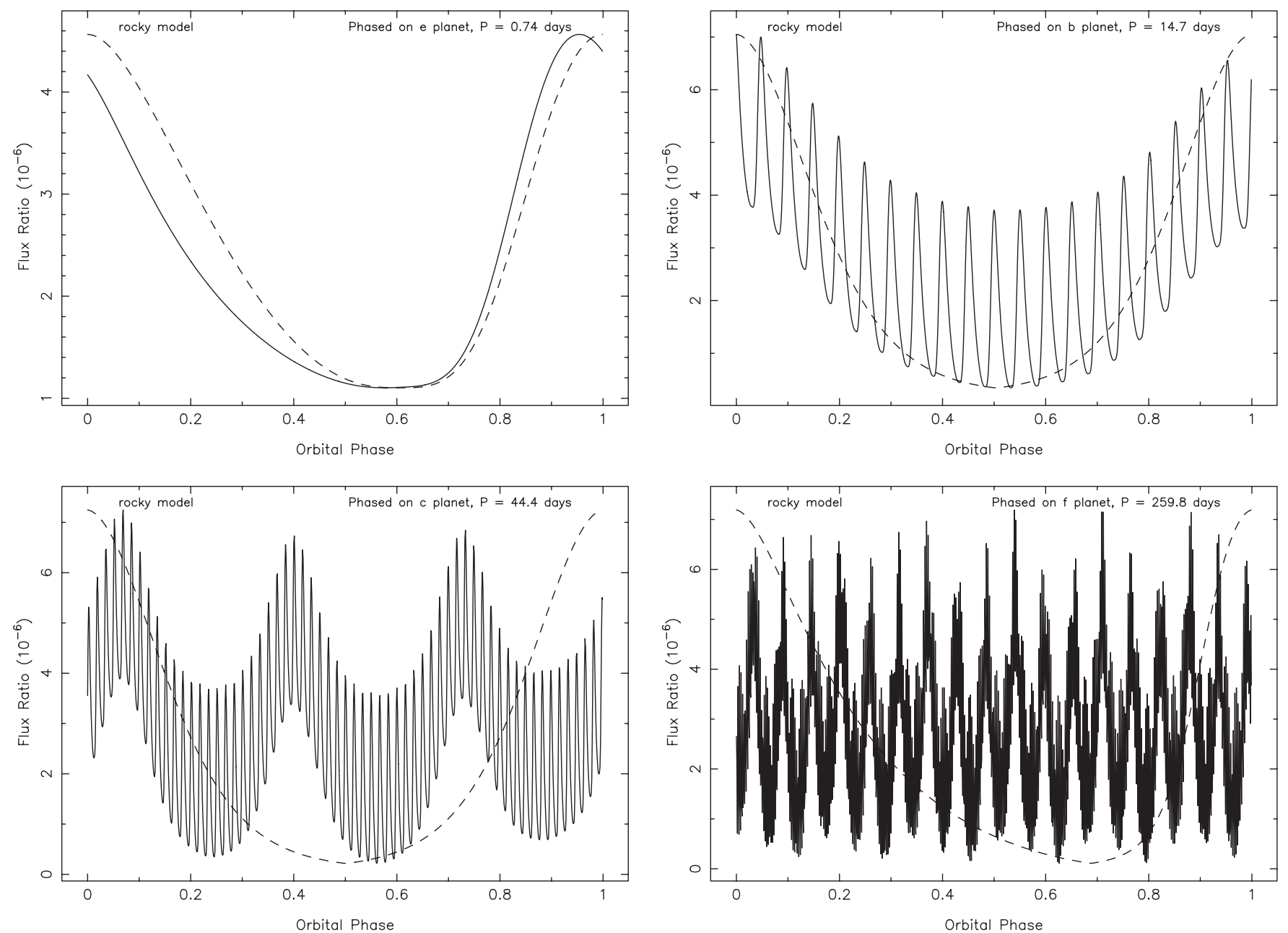

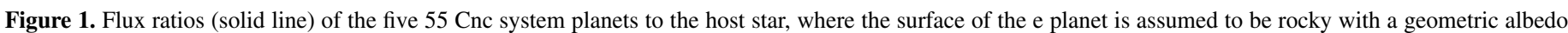

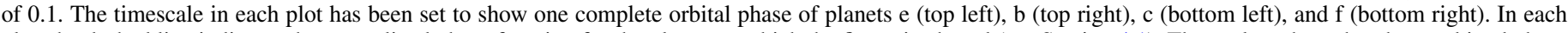

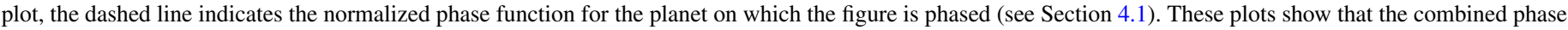

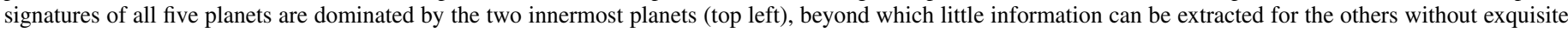
photometric precision.

\subsection{Rocky Surface Model}

Given the size, mass, and density of the planet e, as well as its extreme proximity to the host star, it is likely that any atmosphere that may once have existed has since evaporated and been stripped away by the stellar irradiation. We thus first consider the case that the surface of the planet is of a rocky form, similar to the surface of Mercury. The density of the planet implies a different composition to Mercury (indeed to all the solar terrestrial planets), but the heavier materials likely reside closer to the center of the planet than the surface.

Shown in Figure 1 are the expected phase signatures of the 55 Cnc system using the rocky surface model for the planet e, where the flux ratio refers to the combined flux of all the planets to that of the host star. Hence the phase variation effects for all planets are included in each panel, but panels are zoomed-in on the orbital phase of the e, b, c, and f planets, respectively, and are thus phased on those particular planets in each case. In all four panels, the dashed line corresponds to the phase function of the planet on which the figure is phased. The phase function as shown has been normalized to the $y$-scale of each plot to show the time-dependent contribution of the planet to the total phase curve. For the purposes of this simulation, we first assume that all planets are located at periastron passage, then we move time forward to start the phase curve where the outermost planet in each plot is located at a phase angle of zero. This can be seen in the phase function shown in each panel which is at maximum value at an orbital phase of zero. Therefore these simulations represent a specific orbital configuration, the effect of which we discuss further in Section 4.4.

The phase curves in Figure 1 show that the total phase variation of the system is dominated by planets $e$ and $b$ whose independent flux ratio amplitudes are almost identical; $3.4 \times 10^{-6}$ and $3.3 \times 10^{-6}$, respectively. The flux ratio amplitude of the c planet is almost an order of magnitude less: $7.0 \times 10^{-7}$. Note the asymmetric modulation for the e and f planets (topleft and bottom-right panels) due to their respective eccentric orbits. The maximum flux ratio amplitude of the $f$ planet is $1.1 \times 10^{-7}$ and occurs near the periastron passage near phase 0.7. The outermost planet, $d$, is sufficiently far away from the host star that it contributes a negligible amount of flux to the total flux ratio: $4.0 \times 10^{-9}$. These results are summarized in Table 1.

\subsection{Molten Surface Model}

Winn et al. (2011) estimate a surface temperature of $2800 \mathrm{~K}$ at the substellar point if the planet is tidally locked. Even if the 

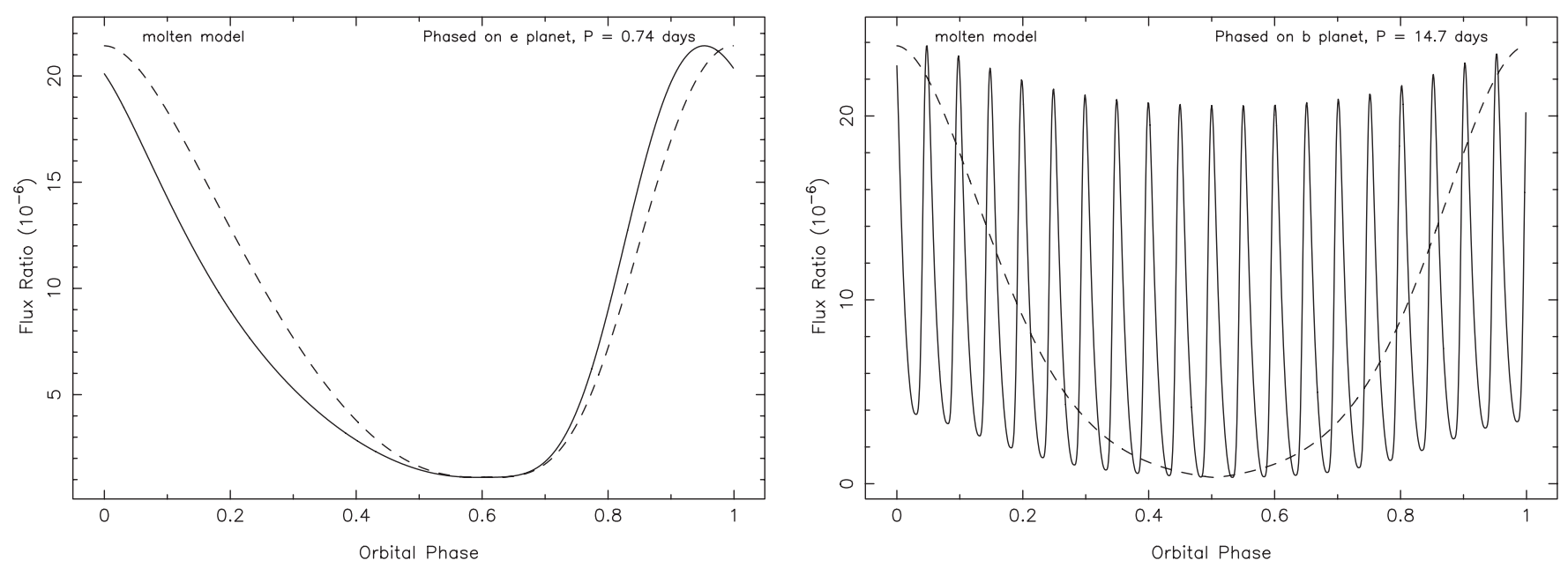

Figure 2. As for the top two panels of Figure 1, but the e planet is now assumed to have a molten surface with a geometric albedo of 0.6 (see Section 4.2).
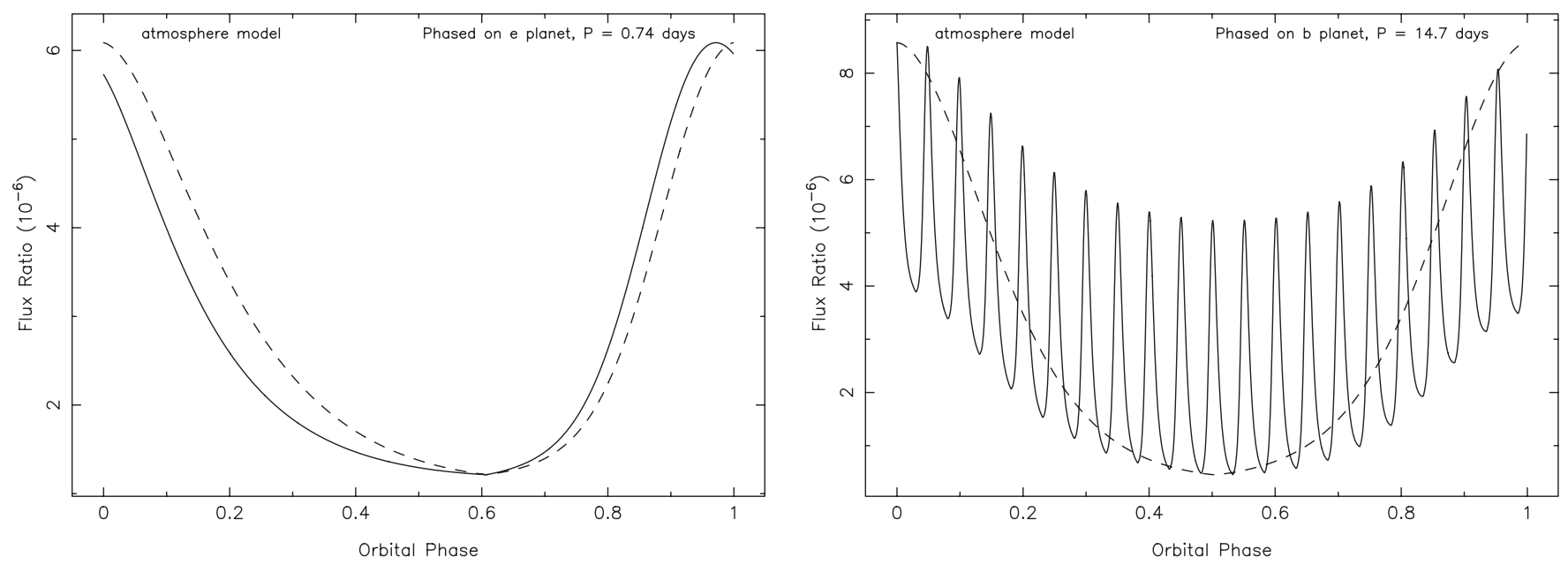

Figure 3. As for the top two panels of Figure 1, but the e planet is now assumed to have an atmosphere with non-isotropic scattering properties (see Section 4.3).

heat is somehow redistributed, the equilibrium temperature will still be as high as $1980 \mathrm{~K}$. This is well above the melting point for igneous rock. In addition, the best-fit solution by Dawson \& Fabrycky (2010) contains a non-zero eccentricity for planet e that would create a "super-Io" effect, as described by Barnes et al. (2010). We thus here consider the entirely plausible case of a molten surface for the inner planet.

Figure 2 is equivalent to the top two panels of Figure 1 in that they are zoomed-in to the phases of planets e and $b$. The much higher albedo causes the e planet to become dominant in the phase curve shown in the right panel (note the different ordinate scales). The e planet now has a flux ratio amplitude of $2.0 \times 10^{-5}$, which is almost an order of magnitude higher than that for the b planet.

\subsection{Atmosphere Model}

The e planet is unlikely to harbor an atmosphere under the extreme conditions of its environment (Winn et al. 2011). Here we consider this possibility for completeness and as a direct comparison with the other two presented scenarios for the surface of planet e. In this case we use the Hilton rather than the Lambert phase function to represent the nonisotropic scattering of the atmosphere. The non-uniform incident stellar flux described in Section 3.5 is insufficient to allow a non-uniform albedo because the reflective condensates will be removed from the upper atmosphere regardless of latitude under such extreme temperatures. As shown in Table 1, the mean geometric albedo for the atmosphere model is only slightly higher than that for the rocky surface due to these reflective condensates being effectively removed.

In Figure 3 we see the combined calculated flux ratio zoomed to the phases of the e and b planets where, once again, the ordinate scales have increased relative to Figure 1. This model produces a flux ratio amplitude for planet e of $4.9 \times 10^{-6}$ which slightly exceeds the amplitude expected from the b planet. We discuss being able to distinguish between this model and the rocky surface model in Section 5.

\subsection{Orbital Configurations and Phase Modulation}

The simulation results provided above describe a specific starting configuration for the planets, which is based upon their periastron passages and phase angles. However, for observations at some random epoch, this configuration will be arbitrary in nature and the phase curves shown here will not necessarily match that which is observed. In particular, the relative phases of the two planets, which contribute the bulk of the total reflected light, $e$ and $b$, will result in phase modulations. Thus there will be optimal orbital configurations for which to search for the phase signatures. The maximum amplitude of the flux ratio will approximately occur where both of the 
planets are simultaneously close to zero phase angle. However, the extraction of only the phase information for planet e will be best achieved when planet $b$ is near inferior conjunction. Fortunately, the orbital periods of these two planets are relatively short, which allows the scheduling of such optimal observating times to be straightforward.

If one requires both high signal-to-noise throughout the measurement, and folding of multiple orbits of planet e, then one needs to be mindful of the contributions of the outer planets as they progress in their orbits. For each orbit of planet e, the outer planets will shift in phase by $0.05,0.017,0.0028$, and 0.00014 , respectively. Through a single orbit this will have minor effects on the total phase variation, but over multiple orbits, will begin to show modulation effects which will dampen the signature of planet e, thus resulting in an incorrect estimate of the flux ratio amplitude. Deriving accurate ephemerides through further RV measurements will help to curtail such effects in photometric monitoring of the phase variations.

\section{SIGNAL DETECTABILITY}

Here we assess the detectability of the planetary flux ratios and the possible effects of stellar variability.

\subsection{Instrumentation Requirements}

From the expected phase amplitude of the two innermost planets of $55 \mathrm{Cnc}$, the instrumentation requirement for successful detection of the phase signatures is photometry with a relative photometric precision of at least $\sim 10^{-6}$. For the e and $b$ planetary phase variations, long-term stability of high-precision photometry is not required. The Kepler mission is already achieving this precision for significantly fainter stars, though it should be noted that the exquisite photometers of Kepler were designed to perform such a task. An example of the high-standard of Kepler precision is the detection of phase and ellipsoidal variations by Welsh et al. (2010) using only the Q1 Kepler data, where the amplitude of this variation is $3.7 \times 10^{-5}$. Using the STIS instrument on the Hubble Space Telescope, Brown et al. (2001) obtained a precision of $1.1 \times 10^{-4}$ per $60 \mathrm{~s}$ integration observation during primary transits of HD 209458b. Such high time resolution is not required for phase variation observations and so binning these data would improve the rms scatter. A future mission that would allow such observations of $55 \mathrm{Cnc}$ to be carried out is the James Webb Space Telescope with the NIRCam instrument, though the wavelength range of this instrument would include a substantial thermal component of the phase variation that would need to be accounted for. The minimum precision mentioned above would adequately confirm or rule out a molten surface for planet e. Further RV characterization of the orbits for the $55 \mathrm{Cnc}$ planets will allow one to accurately predict both the amplitude of the predicted phase signature and times of maximum and minimum flux ratios. This knowledge will help to distinguish the phase signatures from instrumental drift effects.

Future possibilities also exist from the ground, although one needs to also contend with the offsets from night-to-night variations. There are several large telescopes under development that are capable of meeting the challenge of very high photometric precision, such as the European Extremely Large Telescope, the Thirty Meter Telescope, and the Giant Magellan Telescope. It has also been demonstrated by Colón et al. (2010) that precision photometry of $<0.05 \%$ can be achieved with the $10.4 \mathrm{~m}$ Gran Telescopio Canarias through the use of narrowband filters. These was conducted for observing the signatures of known transiting planets which is possible to achieve within a single night. Longer term monitoring of phase signatures will require careful accounting for the aforementioned nightly variations in addition to the air-mass corrections throughout a night.

\subsection{Stellar Variability of 55 Cnc}

If one is able to accomplish the required level of photometric precision, the greatest impediment to studying the planetary phase variations will be the intrinsic stellar variability. An analysis of Kepler data by Ciardi et al. (2011) found that most dwarf stars are stable down to the precision of the Kepler spacecraft, with $\mathrm{G}$ dwarfs being the most stable of the studied spectral types. The main cause of photometric variability in $\mathrm{F}-\mathrm{G}-\mathrm{K}$ stars is starspots and rotation. The rotation period for $55 \mathrm{Cnc}$ has been measured on numerous occasions through photometric variations. Simpson et al. (2010) calculate a rotation period of 44.1 days and Fischer et al. (2008) measure a rotation period of 44.7 days. Winn et al. (2011) also observed variation of the order $10^{-4}$, which is assumed to be the result of both stellar activity and rotation. For the c planet, where the orbital period is close to the rotation period of the star, the variation due to phase and rotation may be difficult to disentangle. The peaks in the power spectrum from a Fourier analysis of the photometry may separate to a degree where the starspot variability can be isolated from the phase signature. The known phase of the planet from the RV analysis will be the greatest aid in discriminating these two signals. It should also be noted that there is an $\mathbf{M}$ dwarf binary companion, $55 \mathrm{Cnc} \mathrm{B}$, with an angular separation of $84^{\prime \prime} .7$ (1150 AU) (Mugrauer et al. 2006), so it is unlikely to be inside a photometric aperture.

\section{CONCLUSIONS}

The multiple-planet system of $55 \mathrm{Cnc}$ presents many opportunities to understand and characterize a system around a solar analog with very different characteristics to our own system. The discovery that the inner-planet transits the host star enhances the opportunities since we can place greater constraints on experiments designs to investigate these properties. Here we have specifically addressed the method of detecting the phase variations of the planets. These results show that the inner two planets have flux ratio amplitudes that are comparable to what has already been detected by Kepler around much fainter stars and will be accessible to next-generation ground and space-based observing platforms if not sooner. Which of the two planets dominates the phase signature depends on whether the planet has a rocky or molten surface, with the molten surface model producing a dominance of planet e to the signature due to the higher reflective properties of the surface. The phase variation of the e planet for a rocky surface is almost indistinguishable from that of an atmosphere model at the level of the precision requirements for detection, $10^{-6}$. These two models have very similar surface albedos and it makes little difference whether one assumes isotropic or non-isotropic (cloud) scattering at such small star-planet separations. The outer planets do not present a significant impediment for the detection of the $\mathrm{e}$ and $\mathrm{b}$ phase variations but one needs to be aware of the expected phase modulations if multiple-phase observations are undertaken to boost signal-to-noise. We have not considered here the thermal component of the flux from the e planet, whose variation could be significant if indeed the planet is tidally locked as suggested by Winn et al. (2011). 
Even though the transit depth is relatively small, the high signal-to-noise possible from transiting planets such as $55 \mathrm{Cnc}$ e demonstrate the value of such objects. The best hope then for more such transiting planets around bright stars lies through investigation of known exoplanets discovered using the RV technique (Kane et al. 2009). Projects such as the Transit Ephemeris Refinement and Monitoring Survey seek to accomplish just that, which will hopefully lead to further characterization opportunities.

The authors thank Alan Boss for insightful discussions as well as Brice-Olivier Demory and Joshua Winn for their useful inputs. We thank the anonymous referee, whose comments greatly improved the quality of the paper.

\section{REFERENCES}

Barnes, R., Raymond, S. N., Greenberg, R., Jackson, B., \& Kaib, N. A. 2010, ApJ, 709, L95

Bean, J. L., \& Seifahrt, A. 2009, A\&A, 496, 249

Bodenheimer, P., Laughlin, G., \& Lin, D. N. C. 2003, ApJ, 592, 555

Brown, T. M., Charbonneau, D., Gilliland, R. L., Noyes, R. W., \& Burrows, A. 2001, ApJ, 552, 699

Butler, R. P., Marcy, G. W., Williams, E., Hauser, H., \& Shirts, P. 1997, ApJ, 474, L115

Ciardi, D. R., von Braun, K., Bryden, G., et al. 2011, AJ, 141, 108

Colón, K. D., Ford, E. B., Lee, B., Mahadevan, S., \& Blake, C. H. 2010, MNRAS, 408, 1494

Cowan, N. B., \& Agol, E. 2011, ApJ, 729, 54

Dawson, R. I., \& Fabrycky, D. C. 2010, ApJ, 722, 937
Deming, D., Knutson, H., Agol, E., et al. 2011, ApJ, 726, 95

Demory, B.-O., et al. 2011a, A\&A, submitted (arXiv:1105.0415)

Demory, B.-O., Seager, S., Madhusudhan, N., et al. 2011b, ApJ, 735, L12

Fischer, D. A., Marcy, G. W., Butler, R. P., et al. 2008, ApJ, 675, 790

Guillochon, J., Ramirez-Ruiz, E., \& Lin, D. 2011, ApJ, 732, 74

Hilton, J. L. 1992, in Explanatory Supplement to the Astronomical Almanac, ed. P. K. Seidelmann (Mill Valley, CA: University Science Books), 383

Kane, S. R., \& Gelino, D. M. 2010, ApJ, 724, 818

Kane, S. R., \& Gelino, D. M. 2011, ApJ, 729, 74

Kane, S. R., Mahadevan, S., von Braun, K., Laughlin, G., \& Ciardi, D. R. 2009, PASP, 121, 1386

Kane, S. R., \& von Braun, K. 2008, ApJ, 689, 492

Knutson, H. A., Charbonneau, D., Cowan, N. B., et al. 2009a, ApJ, 690, 822

Knutson, H. A., Charbonneau, D., Cowan, N. B., et al. 2009b, ApJ, 703, 769

Latham, D. W., Rowe, J. F., Quinn, S. N., et al. 2011, ApJ, 732, L24

Lissauer, J. J., Fabrycky, D. C., Ford, E. B., et al. 2011, Nature, 470, 53

Lovis, C., Ségransan, D., Mayor, M., et al. 2011, A\&A, 528, 112

Malmberg, D., Davies, M. B., \& Heggie, D. C. 2011, MNRAS, 411, 859

Marcy, G. W., Butler, R. P., Fischer, D. A., et al. 2002, ApJ, 581, 1375

McArthur, B. E., Endl, M., Cochran, W. D., et al. 2004, ApJ, 614, L81

Mugrauer, M., Neuhäuser, R., Mazeh, T., et al. 2006, Astron. Nachr., 327, 321

Simonelli, D. P., Dodd, C., \& Veverka, J. 2001, J. Geophys. Res., 106, 33241

Simpson, E. K., Baliunas, S. L., Henry, G. W., \& Watson, C. A. 2010, MNRAS, 408, 1666

Snellen, I. A. G., de Mooij, E. J. W., \& Albrecht, S. 2009, Nature, 459, 543

Sudarsky, D., Burrows, A., Hubeny, I., \& Li, A. 2005, ApJ, 627, 520

Tremaine, S., \& Dong, S. 2011, AJ, submitted (arXiv:1106.5403)

Valenti, J. A., \& Fischer, D. A. 2005, ApJS, 159, 141

von Braun, K., Boyajian, T. S., ten Brummelaar, T. A., et al. 2011, ApJ, 740, 49

Walker, G., Matthews, J., Kuschnig, R., et al. 2003, PASP, 115, 1023

Welsh, W. F., Orosz, J. A., Seager, S., et al. 2010, ApJ, 713, L145

Winn, J. N., Matthews, J. M., Dawson, R. I., et al. 2011, ApJ, 737, L18 